\title{
AMBIENTES PROTECTORES, SEGUROS Y POTENCIADORES DEL DESARROLLO EN LA COMUNA 4 DEL MUNICIPIO DE BELLO, ANTIOQUIA
}

\section{PROTECTIVE, SAFE AND DEVELOPMENT ENVIRONMENTS IN COMMUNE 4 OF THE MUNICIPALITY OF BELLO, ANTIOQUIA}

Yancely Gómez Parra

Catalina Gallego Tobón

Jennyfer Naranjo Loaiza

Luz Enith Sierra Lopera

\section{Sebastián Salazar Galeano}

\section{Alina Gómez Flórez}

Universidad de San Buenaventura, Medellin

\section{RESUMEN}

Se abordan los derechos de los niños desde la educación, la salud y la nutrición, en relación con los espacios públicos en el municipio de Bello (Departamento de Antioquia, Colombia). Se resalta la participación del adulto como actor clave en el acercamiento del niño a los espacios públicos, garantizando las condiciones necesarias para su desarrollo integral, mediante el diseño e implementación de una intervención en el desarrollo urbano y social, que promueva ambientes más seguros, protectores y potenciadores para el desarrollo de los niños, sus familias y la comunidad. Si bien son muchos los esfuerzos de sensibilización y visualización frente al tema, aún existe un desconocimiento por parte de los ciudadanos frente a la necesidad e importancia de generar espacios pensados para los niños y las niñas 
de la ciudad, evidenciando en el municipio poca inversión, planeación y construcción de espacios físicos públicos pensados para la infancia, acarreando un sinnúmero de contradicciones frente a las políticas de infancia y la realidad de los niños en el tema los espacios públicos de ciudad, evidenciando una preocupante carencia de los mismos, ya que, aunque el espacio exista físicamente y los proyectos de desarrollo urbanístico apunten hacia su uso eficiente, la realidad apunta hacia la subutilización e invisibilización de los mismos. En este sentido, la ciudad debe ser construida desde la reflexión constante de infancia y el lugar que ocupa esta en nuestro medio, y así poder garantizar espacios protectores y garantes de un desarrollo infantil de manera plena.

PALABRAS CLAVES: Infancia, Protección de la infancia, Derechos del niño, Espacio de juegos, Espacio urbano, Planificación urbana, Desarrollo del niño.

\section{ABSTRACT}

Children rights are approaches from education, health and nutrition related with public places of Bello township (Antioquia, Colombia). It underlines the participation of adult as an important actor in children's approach to public places, guaranteeing necessary conditions for integral development through design and implementation of an intervention in social and urban development. It promotes safer, protective, potential environments for children, family and community development. While, there are many sensibilization efforts and visualization about the topic, citizens still have ignorance concerning to need and importance to generate places designed for boys and girls of the city, it shows a pour inversion, planning and construction of public places designed for children in the town. It carries a countless contradictions facing childhood policies and children reality in city's public places, evidencing a worrying lack of them, as the place exists in a physic way and urban development projects that point to efficient use; the reality points to underutilization and invisibility of them. In that sense, city must be built on the constante reflection of childhood and the space it takes over in our environment, in this way, and thus be able to guarantee protector guarantor places of childhood fully development.

Keywords: Child Safety, Child Welfare, Child protection services, Protective factors, child development, Children's rights, City planning, Child protection.

\section{INTRODUCCIÓN}

Son numerosos los abordajes que se han realizado en el marco de los ambientes potencializadores, seguros y protectores, los cuales se han soportado a partir de los aportes teóricos sobre la importancia que tienen, de manera que puedan generar experiencias significativas a través de la interacción con diferentes materiales, personas y lugares. Los ambientes protectores se pueden formar en diversos espacios en el que se fomenta la socialización, el cuidado, el afecto y el respeto por la opinión y la integralidad de los demás, teniendo como objetivo formar seres autónomos y seguros de sí mismos, donde puedan tomar decisiones libremente, dejando las inseguridades y dudas que los rodea. Los niños se interesan constantemente, en pleno proceso de desarrollo, en experimentar el medio; por lo tanto, es fundamental implementar espacios y materiales didácticos, en los cuales los niños tengan la posibilidad de interactuar de manera libre y creativa: En los espacios seguros se pretende brindar diversos materiales y escenarios adecuados para un desarrollo sano y libre de peligro.

Al abordar el tema de un ambiente educativo no solo se refiere a un aula de clase, hay numerosos espacios como parques, bibliotecas o espacios deportivos que son generadores 
de aprendizajes, en los cuales se puede potencializar el conocimiento significativo de los niños y niñas, en donde los agentes educativos y cuidadores son generadores y motivadores de formar espacios de interés para la niñez. Igualmente, los espacios públicos y privados han sido configurados por los habitantes de la misma sociedad, la cual se ha encargado de clasificar y determinar cuál es la utilidad que se le debe dar a cada uno de estos, delimitándolo de tal manera que no son tomados en cuenta como posibles escenarios pedagógicos en las distintas áreas del conocimiento. En consecuencia, surge la necesidad de generar ambientes de discusión pública sobre la importancia de la sensibilización en la Comuna 4 del municipio de Bello, implementándose en jardines infantiles, espacios públicos y cerrados, y así determinar el impacto que esto puede generar en la comunidad y agentes educativos, padres de familia y cuidadores, de manera que se puedan lograr cambios significativos en lugares diferentes al aula de clase. Existen diversos espacios donde se puede realizar estos procesos de aprendizaje, para que generen interés y curiosidad en la infancia, procesos acompañados por padres, ciudadanos y agentes educativos que están en constante interacción con los niños y niñas.

En las últimas décadas, los niños y niñas han sido educados y se han desarrollado en espacios que no son completamente favorables, a diferencia de los padres y abuelos, que crecieron en espacios donde podían encontrarse con ellos mismos y con sus pares. Por otro lado, en Colombia, se evidencia que diversas organizaciones han tomado conciencia sobre la importancia de implementar espacios para generar en los niños y niñas ambientes protectores y seguros, especialmente con mayor rigor en zonas del país en donde la violencia juega un papel principal. Así, pues, se identifican en el municipio de Bello diferentes espacios brindados por la comunidad, tales como bibliotecas, parques, centros educativos, museos, entre otros, los cuales no han tenido en cuenta, al momento de ser planeados, diseñados y construidos, tres categorías necesarias a la hora de pensar en los niños y niñas como agentes en permanente formación, esto es, ambientes potencializadores del desarrollo, protectores y seguros. Se percibe que la ubicación de estos espacios, en los aspectos de infraestructura, no son adecuadas, el ruido y la polución afectan el desarrollo de los niños y niñas, o se encuentran en zonas de violencia y conflicto, impidiendo, por tanto, que la primera infancia se favorezca en su totalidad.

Se evidencia que tanto los espacios públicos y privados son visitados de manera permanente por los niños y las niñas, es por esto que surge un interrogante acerca de estos ambientes, esto es, si cumplen o no con la característica de ser potencializadores, protectores y seguros, para llevar a cabo en los niños y niñas un adecuado desarrollo. En este sentido, esta investigación permite dar respuesta a la pregunta planteada $\mathrm{y}$, posteriormente, intervenir en la comunidad con el fin de sensibilizar y promover que estos espacios sean propicios para el desarrollo de los niños y niñas, a la vez que cumplan con las características adecuadas para satisfacer las necesidades de la primera infancia. Por consiguiente, se evidencia la necesidad de indagar autores e investigaciones realizadas de manera internacional, nacional y local, con el objetivo de poder intervenir en la comunidad y realizar transformaciones sociales sobre la importancia de establecer espacios potencializadores, protectores y seguros para apoyar el desarrollo los niños y niñas. Esta investigación se lleva a cabo en la Comuna 4 del municipio de Bello, a través de diferentes instrumentos de recolección de información. Igualmente, se implementa una guía metodológica $y$, finalmente, se realiza una medición sobre el impacto generado en la comunidad. 


\section{METOdOLOGÍA}

Para la realización de este estudio, se hace indispensable la utilización de la metodología mixta. En lo que respecta a lo cuantitativo, la investigación se apoya dentro de los estudios no experimentales-descriptivos, ya que es pertinente describir el estado en que se encuentran los diferentes espacios en cuanto a seguridad, protección y potenciador de desarrollo en los niños de 0 a 6 años en la Comuna 4 del municipio de Bello, con una muestra de 73 niños de instituciones públicas y privadas y 102 adultos de dicha comuna, lo que permite explorar un panorama frente a las diferentes realidades que allí se viven. Con respecto a la investigación cualitativa, y gracias a su carácter flexible, se apuesta por un diseño hermenéutico, el cual se interesa por comprender esos espacios desde lo seguro, lo protector y lo potenciador del desarrollo. Se plantea el abordaje de técnicas como la aplicación de encuestas, entrevistas, revisión documental, cartografía social e instrumentos como cuestionarios, guías de entrevistas, que son determinantes para llevar a cabo dicho proceso. En primera instancia, se realiza una caracterización usando diferentes instrumentos de recolección para obtener información de fuentes primarias y secundarias, con la cual se identifican las condiciones actuales del espacio público del barrio seleccionado. Se tienen en cuenta algunas condiciones de seguridad, sostenibilidad y protección que benefician la participación y el desarrollo integral de los niños, las familias y la comunidad.

La información obtenida es de carácter cualitativo y cuantitativo, la cual es procesada y analizada usando herramientas de análisis mixto, lo que permite tener en cuenta todas las voces de la población objetivo. Luego del procesamiento y el análisis de los resultados, se diseña una propuesta metodológica que involucra estrategias de intervención urbana y social, donde se pretende un mejoramiento del espacio y, además, un involucramiento de la comunidad en la apropiación y la protección de los espacios públicos. Finalmente, se calculan los indicadores que permiten evaluar la intervención y las estrategias planteadas. Los indicadores se diseñan para medir la seguridad, la sostenibilidad y la protección, que pueden ser comparables con la información recogida en la caracterización (línea base) para, posteriormente, evaluar resultados e impacto. 


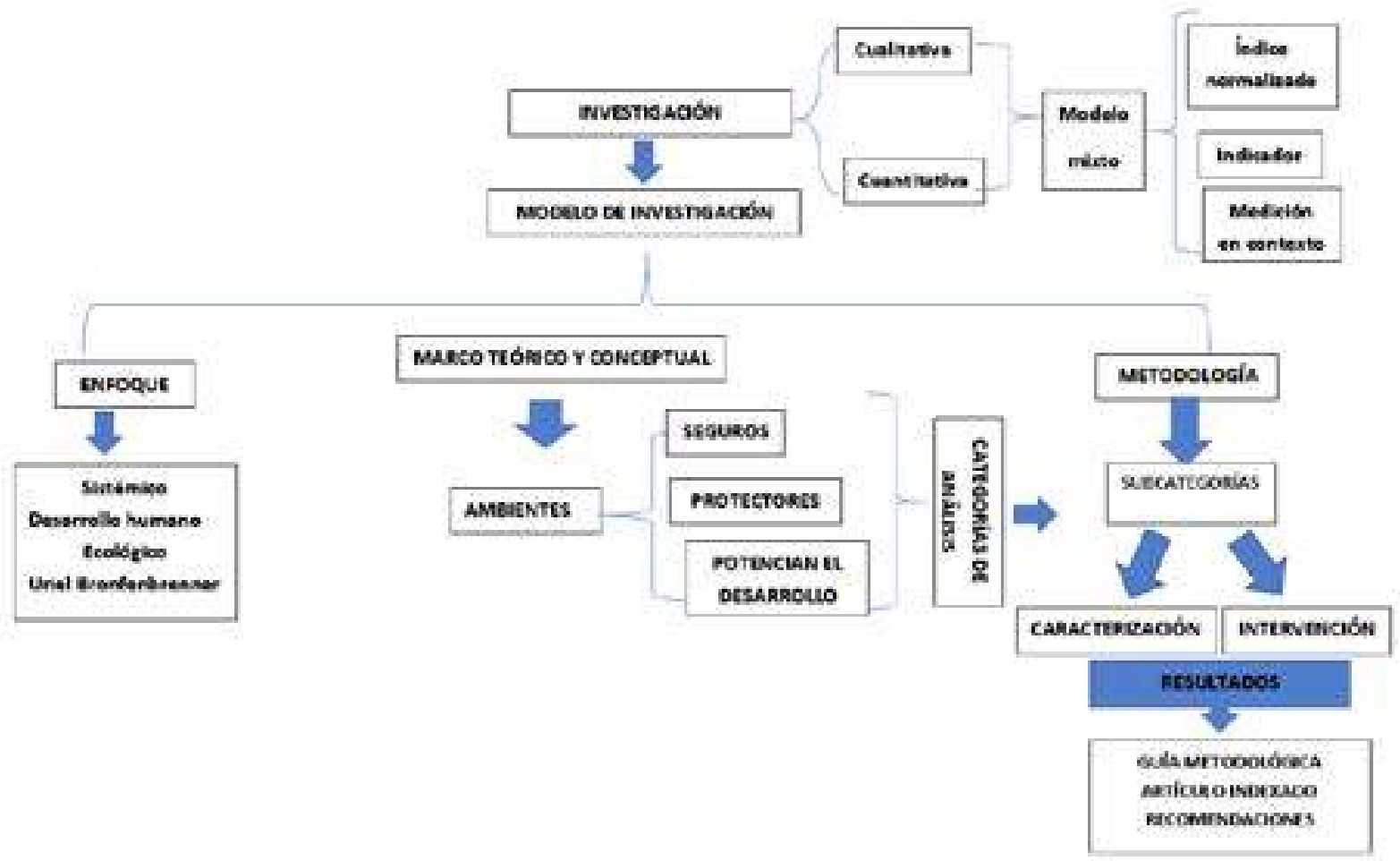

Figura 1. Diseño metodológico.

\section{RESULTADOS}

\section{Espacios públicos pensados para la infancia. Categorías de análisis de los ambientes}

El espacio púbico comprende una definición multidimensional que abarca aspectos físicos, territoriales, políticos, sociales, económicos y culturales, por lo que para su comprensión se debe tener presente que incluye escenarios donde se ejerce el derecho a la libre locomoción, definido en el Artículo 24 de la Constitución Política (Colombia. Asamblea Nacional Constituyente, 1991), ya sea en espacios abiertos como calles, parques y plazas o en recintos cerrados como bibliotecas, centros comunales, entre otros, por lo que el espacio público se reconoce desde su múltiple dimensionalidad (García Vázquez, 2013). Con el fin de recolectar la percepción de los adultos respecto a los espacios públicos del municipio de Bello, se realiza una encuesta donde se indagan tres categorías básicas sobre la percepción, la protección, la seguridad y lo potenciador del desarrollo de los ambientes de la Comuna 4 de Bello, lo cual arroja como resultado que el $66 \%$ de la población encuestada reconoce el parque principal como un espacio público dentro de la comuna. Por su parte, el espacio menos reconocido por la población encuestada es el Comando de Acción Inmediata (CAI) de la Policía Nacional, con un $21 \%$ de percepción. No es de extrañar que el espacio público con mayor reconocimiento sea el parque (plaza) central, ya que es un lugar de encuentro, ubicado cerca al comercio y a los centros religiosos; además, es uno de los lugares más frecuentados por los adultos mayores.

La iglesia y el parque son los lugares públicos de la Comuna 4 de Bello con mayor nivel de referenciación por parte de los niños encuestados, con un $29 \%$. Lo anterior brinda a la investigación validez interna, ya que al triangular los datos es posible establecer que la respuesta coincide con la de los adultos intervenidos. 


\section{ESPACIO PÚBLICO RECONOCIBLE}

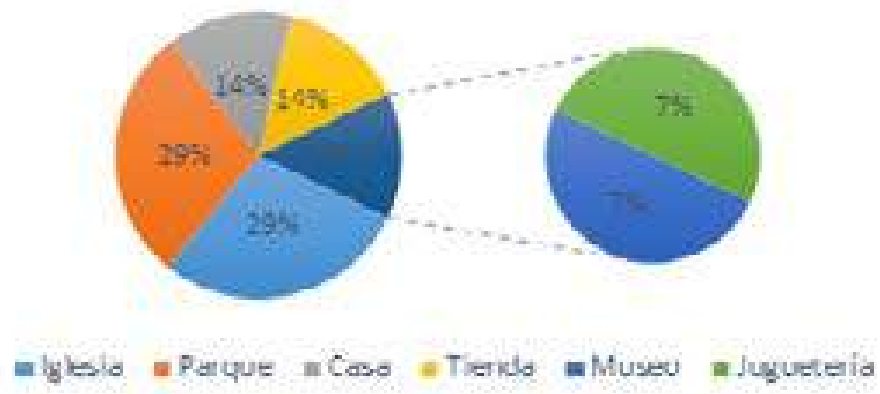

Figura 2. Espacio público reconocible.

Fuente propia, voces de los niños.

El acceso a un espacio público de calidad es uno de los ejes centrales del derecho a la ciudadanía, pues propone una dimensión política del espacio público; así como el espacio público se configura en un espacio dialógico entre la administración pública y la ciudadanía, la primera faculta el dominio del suelo, garantizando su uso, y la segunda le otorga el carácter de dominio público (ejerciendo un uso real), lo que se denomina como la apropiación cultural colectiva (Borja, 2003; García Vázquez, 2013); en este sentido:

La dinámica que encontremos en cualquier espacio público de la ciudad depende directamente del nivel de apropiación dado por parte de la gente, más que de cuán bien se encuentre equipado el espacio (es decir, la calificación de espacio público es aportada por la gente y no por el planificador) (García Vázquez, 2013, p. 5).

Por su parte, las voces de los niños manifiestan que el tráfico vehicular es un gran inconveniente para su seguridad en el espacio público, en tanto se identifica que la velocidad vehicular y la contaminación auditiva son factores de riesgo, el primero con un $86 \%$ y el segundo con un $71 \%$ de voces encuestadas. En este orden de ideas, los adultos plantean, en un mayor porcentaje, que los espacios públicos no son seguros para los niños, con un $76.9 \%$, y expresan, además, que debido a los altos niveles de violencia, el espacio público de la Comuna 4 no es seguro para la población infantil, con un $32 \%$ de voces encuestadas (Figura 3 ).

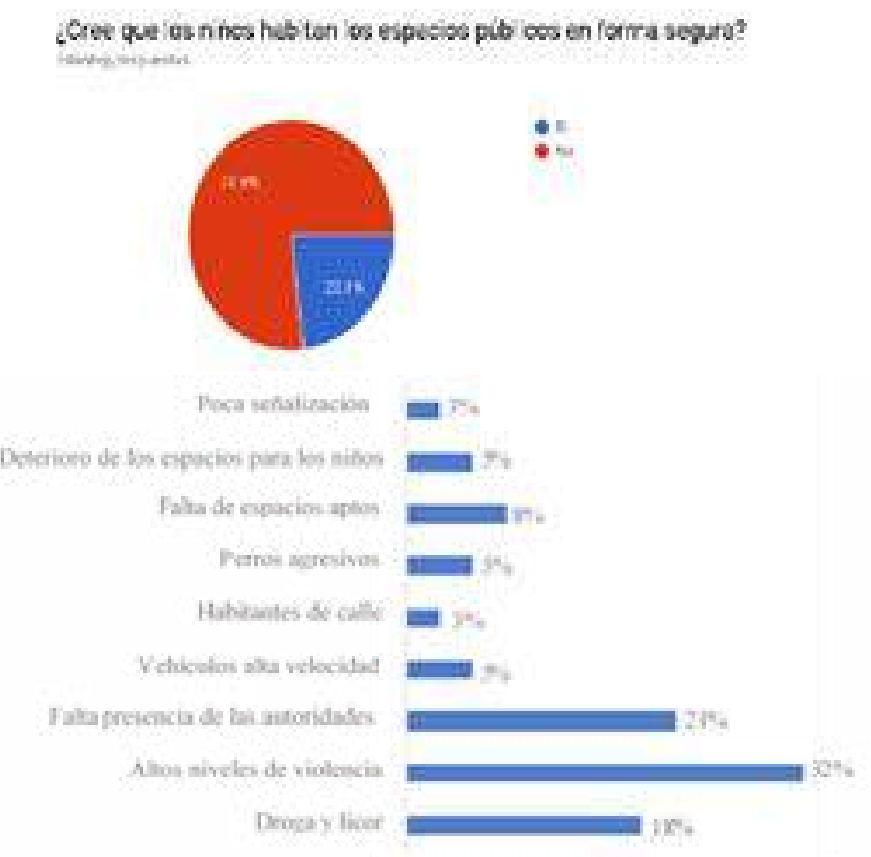

Figura 3. Voces de los niños. 
Sin embargo, para que estás dimensiones del espacio público puedan favorecer a los niños es necesario identificar a la primera infancia como parámetro de las necesidades de todos los ciudadanos, ya que investigaciones recientes, en especial aquellas que están relacionadas con el diseño universal, no tardan en señalar que cuando el espacio público se utiliza como parámetro de referencia, la primera infancia y otras poblaciones se ven favorecidos los adultos mayores, las personas con movilidad reducida y demás población en general.

Figura 4. Espacios públicos más identificados por la comunidad.
En cuanto a los espacios diseñados para el uso exclusivo de los niños (en compañía de sus familias) en la Comuna 4 del municipio de Bello, el parque y el hogar o jardín infantil (CDI) encabezan la lista, con un $46 \%$ de los participantes, seguido por otros espacios, en los cuales se nombra de manera recurrente el Polideportivo Tulio Ospina y los centros de recreación, educación y esparcimiento de la Caja de Compensación Familiar (Comfama), como se evidencia en la Figura 4.

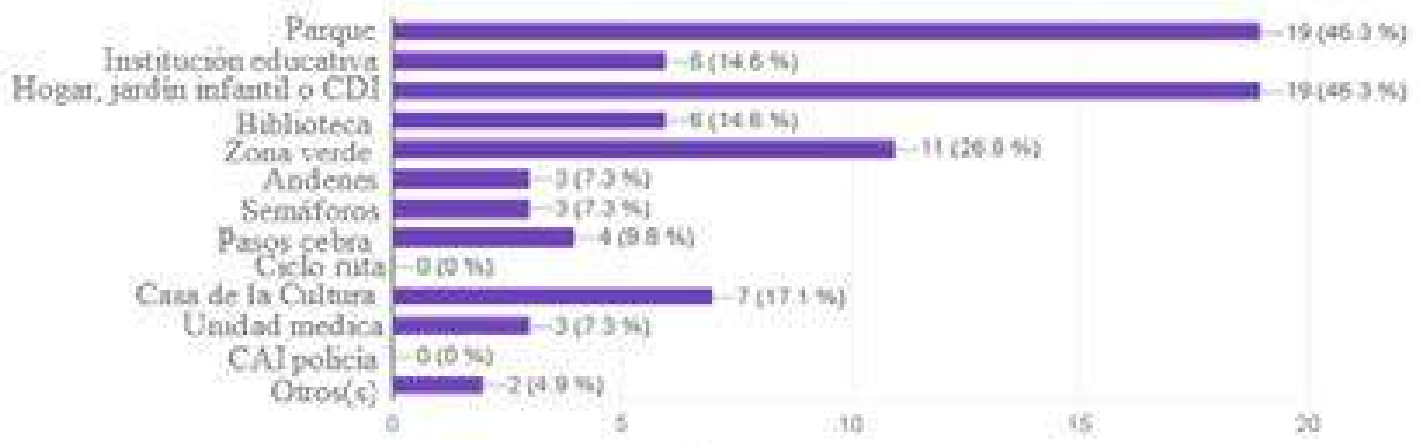

En este sentido, se plantea la crisis de las ciudades bajo el siguiente argumento:

En las últimas décadas, y de manera clamorosa en los últimos cincuenta años, la ciudad, nacida como lugar de encuentro e intercambio, ha descubierto el valor comercial del espacio y ha trastornado todos los conceptos de equilibrio, de bienestar y de convivencia, para cultivar sólo programas a fin de obtener beneficios. Se ha pensado, proyectado y evaluado la ciudad tomando como parámetro un ciudadano medio con las características de adulto, hombre y trabajador, y que corresponde al elector fuerte. De este modo la ciudad ha perdido a los ciudadanos no adultos, no hombres y no trabajadores, ciudadanos de segunda categoría, con menos derechos o sin ellos (Tonucci, 2015, p. 20).

Igualmente, es importante conocer los gustos de los niños frente a dichos espacios públicos, evidenciando que el $42 \%$ indica que el lugar que más disfrutan es el parque.

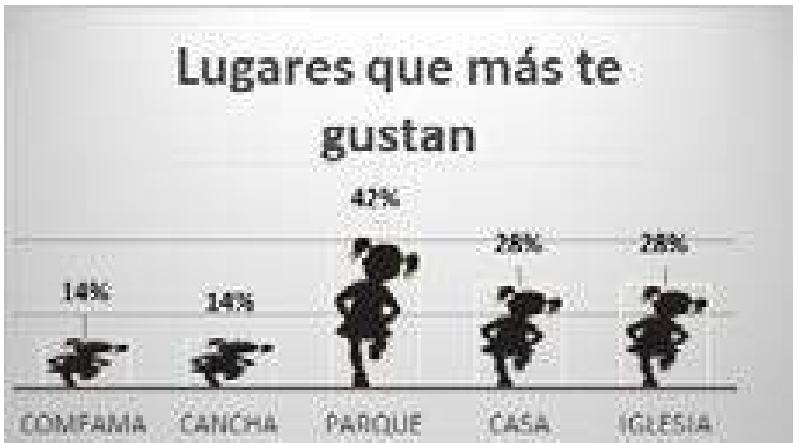

Figura 5. Lugares que más les gustan a los niños de la Comuna 4 del municipio de Bello. 
Teniendo en cuenta lo anteriormente mencionado, es importante referir la relación adyacente entre el espacio público y la primera infancia, compuesta por resistencias (García Vázquez, 2013) de ambientes adultilizados (Malone, 2010; Tonucci, 2015) y, ante todo, una relación compleja (Román Rivas \& Pernas Riaño, 2009). En este sentido, la relación espacio público y primera infancia se da por ausencia del niño en las calles de las ciudades (Román Rivas \& Pernas Riaño, 2009), por las implicaciones que adquiere el concepto callejero en las sociedades modernas (Sánchez Parga, 2004), pues los niños son excluidos del espacio público y luego considerados invasores del mismo (Román Rivas \& Pernas Riaño, 2009), recluidos en las casas y entretenidos con los productos de la sociedad tecnificada (Tonucci, 2015).

En este sentido, la relación primera infancia y espacio público parece divorciada a pesar de que el resurgimiento de la infancia y del urbanismo van de la mano, pues ambas nacen durante el esplendor de la burguesía. Por lo anterior, los niños y los adolescentes se enfrentan a la difícil tarea de aprender a buscar sentido a una ciudad que no les pertenece y que les obliga a hacerse mayores (Román Rivas \& Pernas Riaño, 2009). De otro lado, el urbanismo está compuesto por lo que se denominan "arquitecturas visibles y arquitecturas invisibles" (Caballenas et al., 2005, p. 268), donde las arquitecturas invisibles no solo hacen referencia a la arquitectura que no es fácilmente perceptible por el ojo humano (como es el caso correspondiente al acueducto, los trenes subterráneos, los sótanos, entre otros), sino que en términos de primera infancia está compuesta, a su vez, por la ubicuidad transitoria o permanente de la infancia en el urbanismo, de donde se desprenden los conceptos de lugares perdidos y lugares recuperados para la cultura infantil (Abad Molina, 2014).

\section{AMBIENTES PROTECTORES}

Se define un ambiente protector como "un espacio seguro de participación, expresión y desarrollo para los niños, niñas y adolescentes" (Fondo de las Naciones Unidas para la Infancia [UNICEF], 2019, párr. 3). Bajo estos términos, la organización puntualiza que un ambiente protector está dado por brindar educación de calidad, formación especial y servicios amigables a partir de un enfoque de desarrollo inclusivo, donde se tengan en cuenta grupos poblacionales indígenas, afrodescendientes y migrantes. En esta misma línea, se proponen una conceptualización desde la perspectiva del desarrollo humano, donde es considerado ambiente protector "aquel en el cual los niños, niñas y adolescentes adquieren las mejores herramientas posibles para la construcción de su vida, disfrutando de oportunidades para desarrollar su capacidad individual en un entorno seguro y propicio" (Vertel Betancur \& Cuervo Duque, 2013). Aunque ambas definiciones coinciden en que un ambiente protector tiene como público objetivo la infancia y la adolescencia, se logra ampliar el término hacia la concepción del ciclo vital, postulando que un ambiente protector piensa en los espacios y las condiciones para el crecimiento y el desarrollo desde el periodo de la gestación, lo que incluye tener en cuenta tanto a los niños y adolescentes como las necesidades e intereses de madres, tanto gestantes como lactantes.

En esta categoría, los adultos expresan que los espacios públicos de la Comuna 4 están pensados, principalmente, en un 53\% para garantizar el derecho al juego, como se evidencia en la Figura 6. 


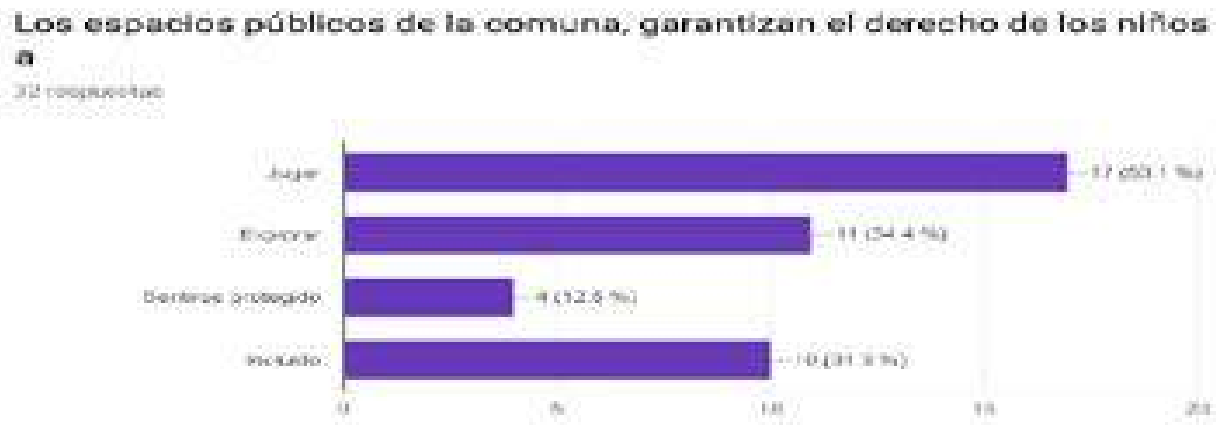

Figura 6. Percepción de los adultos frente a los espacios públicos.

Desde el municipio de Bello se trabajan diferentes estrategias que favorecen los ambientes protectores pensados para la infancia, algunas de ellas enmarcadas en las políticas de apoyo a la niñez y a la adolescencia y hacia los planes de desarrollo para el fortalecimiento de la familia.

De igual forma, se evidencia que las instituciones educativas, los hogares infantiles y los centros de desarrollo integral (CDI) son los espacios públicos con mayor reconocimiento en la prevención de situaciones sociales negativas con los niños, pues cuentan con protocolos para la prevención del cualquier tipo de maltrato infantil y rutas de restablecimiento de derechos.

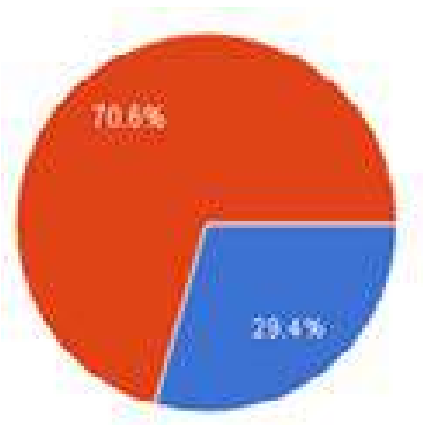

?

Figura 7. Espacios públicos en la Comuna que ayudan en la prevención de situaciones sociales negativas con los niños.
Pinheiro de Almeida (2012) aborda el tema de los espacios públicos donde juegan y participan los niños, donde se intervienen niños, padres de familias, educadores, psicólogos y sociólogos, estableciendo algunos factores de riesgo, de los que cabe señalar los siguientes:

- Los niños tienen cada día menos espacio y tiempo para desarrollar actividades lúdicas.

- La industrialización y la urbanización modifican las costumbres, las maneras de vivir e incluso la estructura familiar, y contribuyen para que muchas actividades de los niños no solo se modifiquen, sino que hasta desaparecen.

- La falta de espacio como consecuencia de la inseguridad en los grandes centros urbanos.

- El cúmulo de actividades extracurriculares de las instituciones educativas, lo que ha ocasionado que los niños pierdan sus compañeros de juego.

- Con el crecimiento de las ciudades, cada día los espacios para los niños son más reducidos.

- La incursión de la mujer en el mundo laboral hace que los niños tengan que permanecer más tiempo en las instituciones y menos tiempo en el espacio público.

La UNESCO, la UNICEF y la ONU (citados por Pinheiro de Almeida, 2012) añaden 
a la lista anterior los siguientes factores de riesgo:

- Planeación ambiental inadecuada, patente en la proporción deshumanizada de las construcciones, formas de viviendas impropias y malas gestiones de tráfico.

- La exposición constante de los niños a la guerra, a la violencia, a la explotación y a la destrucción.

- La valoración de la competición y del ganar a todo coste del deporte infantil.

\section{AMBIENTES POTENCIADORES.}

Según la Comisión Intersectorial de Primera Infancia:

El desarrollo tiene una dinámica irregular, discontinua, de cambios y retrocesos que son vividos por cada niña o cada niño de manera diferente. Las variaciones que se presentan en cada uno, y en relación con otras niñas y niños de la misma edad, manifiestan que, desde el inicio de la vida, los ritmos son propios y cada uno avanza al compás que marca sus características, sus condiciones y las interacciones que realice con las personas significativas que le rodean, en un contexto social y cultural específico (2013, citado por Colombia. Ministerio de Educación Nacional, 2014, p. 15).

En el Documento 10 se definen los ambientes potenciadores del desarrollo en relación con la creación de los espacios educativos significativos para el desarrollo integral de la primera infancia, así como la concepción que considera al espacio como el tercer educador (Colombia. Ministerio de Educación Nacional, 2009). Sin embargo, los procesos de aprendizaje no dependen exclusivamente de los espacios físicos y los materiales, en ellos intervienen las relaciones que se establecen, los procesos de socialización y la comunicación, así como las dinámicas que emergen entre ellos. La articulación de dichos aspectos configura los ambientes potenciadores del desarrollo y se convierten en la puesta en escena del acto educativo.

Con lo anterior se pretende favorecer el desarrollo integral de niños y niñas, partiendo no solo de las actividades que día tras día se realizan en los centros educativos, sino también generando allí un reconocimiento como sujetos activos en la construcción de los aprendizajes que se forjan en la escuela, articulando procesos y ambientes comunicativos, afectivos, cognitivos y sociales en las dinámicas e interacciones de la escuela y ambientes disponibles para favorecer el desarrollo integral de niños y niñas.

Los adultos indican que los niños adquieren aprendizajes culturales (25\%) y educativos (25\%) en el espacio público, seguido por aprendizajes lúdicos (19\%), sociales (13\%) y de exploración (13\%). En consecuencia, los adultos consideran que el desarrollo del aprendizaje de tipo instruccional y académico es el que con mayor frecuencia se adquiere en el espacio público, debido a que su punto de mayor referenciación corresponde a lugares de instrucción académica y consulta, los cuales relacionan con la interiorización de la buena conducta y los modales y el proceso de enseñanza aprendizaje (Figura 8).

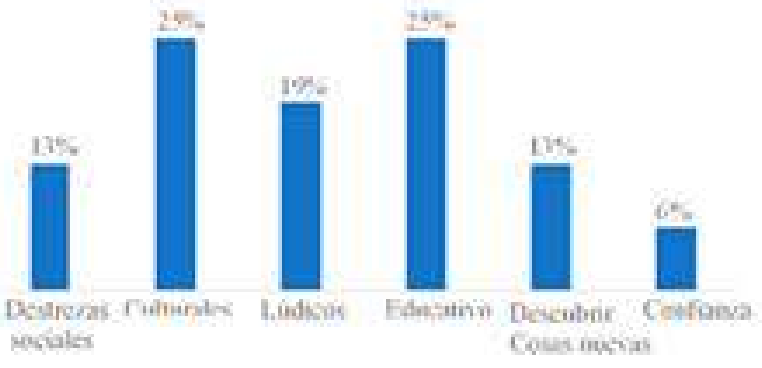

Figura 8. Percepción de los adultos frente a los aprendizajes adquiridos en los espacios públicos. 
Las instituciones educativas son las encargadas de crear ambientes que aporten al desarrollo evolutivo de los sujetos que allí participan; sin embargo, no solo las escuelas son las encargadas de estas construcciones, también las familias, el Estado, los entes administradores locales municipales, las juntas de acción comunal, entre otros, son agentes determinantes para la evolución, esto en un contexto diferenciador, es decir, que hoy se deben articular todas las acciones posibles que intervengan positivamente en lo que se llama el desarrollo humano.

Según el instrumento, los parques (70\%), seguido de los hogares infantiles y los centros de desarrollo (38.2\%), se constituyen en los lugares más frecuentados por los niños. Los espacios abiertos de circulación como andenes, semáforos, pasos peatonales y demás, siguen presentando baja frecuencia en las respuestas proporcionadas.

Al abordar otros ambientes que generan desarrollo evolutivo, es importante mencionar el que se presenta a través de la escuela, pues es allí donde lo social permite un aspecto evolutivo por medio del acercamiento entre los seres humanos; asimismo, el barrio también tiene su aporte al desarrollo potenciador de la infancia mediante los momentos de diversión, de encuentro lúdico; igualmente, la familia es un agente que refuerza el desarrollo integral desde la infancia; por otro lado, la toma de decisiones durante el juego barrial, la competencia, la recreación, entre otros, son actividades que ayudan a orientar, a construir, a formar e integrar una cultura dinamizadora que identifica un ambiente desarrollador para la infancia, esto es, que la vida del barrio desencadena aspectos importantes para la socialización y el fortalecimiento de agentes constructores de vida, pues este es un ambiente vital para el desarrollo humano.
Las voces de los adultos en la encuesta expresan (30\%) que las razones por las cuales los parques, CDI y hogares infantiles son espacios generadores de aprendizaje, ya que en dichos lugares se encuentra un porcentaje más elevado de actividades, tanto pedagógicas como recreativas. En cada ambiente (hogar, jardín, centro infantil, espacios culturales, naturales, recreativos), los niños y las niñas encuentran diferentes propuestas para la exploración e interacción con los materiales y objetos, a su tiempo y ritmo, favoreciendo la comunicación y el aprendizaje. Se propone, entonces, que los ambientes pensados para la infancia deben ser:

- Provocador, en tanto suscita el asombro, la curiosidad, la pregunta, nuevas conexiones, relaciones y elaboraciones desde los saberes previos, resignificando la vida cotidiana (Colombia. Ministerio de Educación Nacional, 2017).

- Retador, que plantea desafíos físicos, motores, sociales, cognitivos y emocionales para asumir tareas cada vez más complejas, activando la creatividad y la autonomía.

- Sensible, que estimula los sentidos y la fantasía a partir de las diversas percepciones sensoriales que ofrece, y de la experiencia concreta en el entorno que habita (Colombia. Ministerio de Educación Nacional, 2017).

- Dialógico, que facilita el encuentro de saberes y la construcción de ciudadanía, que se retroalimentan constantemente en el placer por conocer.

- Integrador, que posibilita el desarrollo armónico de cada niño y niña de acuerdo con sus intereses y el despliegue de todas sus capacidades y habilidades.

- Metafórico, que favorece que la realidad se exprese desde lo poético, lo narrativo y 
lo literario, facilitando con ello su acceso a aprendizajes significativos.

- Incluyente, que considera las necesidades y particularidades de niños y niñas, así como del agente educativo, desde una perspectiva de la diversidad, la diferencia y la multiculturalidad.

Las orientaciones básicas para el diseño de los ambientes potenciadores del desarrollo, requieren de los agentes educativos:

- El diseño de diferentes ambientes con intencionalidad, con sentido lúdico $\mathrm{y}$ artístico, para acoger a los niños y las niñas de diferentes edades e intereses. Cada ambiente debe estar pensado y recreado de una manera cálida, acogedora y con gusto estético, que presente de manera armoniosa colores, formas, texturas y olores; dispuesto con materiales adecuados y de fácil acceso para los niños y las niñas, sin sobresaturación.

- La creación de ambientes que favorecen la inclusión, respetan las elecciones y preferencias con las cuales los niños y las niñas se identifican para disfrutar, interactuar, jugar y aprender a nivel individual y colectivo.

- La incorporación en los ambientes de elementos y expresiones culturales, con los cuales los niños y las niñas se identifican, estableciendo conexiones con las temáticas que recrean la interculturalidad.

- Los niños y las niñas se desplazan libremente hacia lo que les interesa, se deben respetar las improntas que ellos manifiestan frente a pares, objetos, materiales y espacios. No todos los niños y las niñas tienen que estar haciendo lo mismo a la vez, ni tienen que demostrar el mismo interés, unos pueden estar en juego de roles, otros dramatizando, algunos construyendo, pintando, pasando las páginas de un libro, leyendo las imágenes y construyendo historias, entre otras actividades.

- Son necesarios ambientes que favorezcan los lenguajes expresivos como, por ejemplo, juego de roles, de construcción, de expresión artística, entre otros. Se necesitan juguetes simples, materiales de la naturaleza y de desecho, materiales que puedan explorar, transformar y ensamblar. Los elementos también pueden conjugar sonido, movimiento, así como efectos de luz, color y formas.

- Se privilegia el trabajo de los niños y las niñas en pequeños grupos en diversos ambientes, pues permiten los aprendizajes recíprocos en los que pueden planear, discutir y ejecutar actividades conjuntamente, dar a conocer al grupo lo que piensan y sienten y aprender de sus pares y agentes educativos.

- Los agentes educativos están en permanente observación de los gustos, las expresiones, las preguntas, las respuestas y las acciones de los niños y las niñas. Se hace registro de las experiencias y se dispone de un lugar preferencial para las producciones de los niños y las niñas.

- Los ambientes abiertos se deben visualizar desde la perspectiva de ambientes potenciadores del desarrollo, ambientes que permitan actividades de movimiento tanto a nivel grupal como individual; de igual manera, que permitan tener intimidad para el juego en solitario, la conversación, el descanso y la lectura.

- Se garantiza la seguridad física y emocional de los niños y las niñas, haciendo elección de lugares aireados, luminosos, limpios, provistos de los recursos y materiales necesarios.

- La disposición de los materiales, de manera tal que estén al alcance de los niños y las 
niñas, en un orden básico que se conserva al finalizar el juego o la actividad, para lo cual se les debe involucrar en actividades de organización de los espacios, lo que de manera regular contribuye a la construcción del sentido de pertenencia e identidad y a la adquisición de hábitos de aseo y orden.

- La introducción y el retiro de materiales y elementos de los ambientes para renovar y "oxigenar" las interacciones y las propuestas de actividades de acuerdo a las épocas y fiestas del año, a las dinámicas del proyecto de exploración y demás eventos que emergen en la cotidianidad y requieren de otras ambientaciones con el fin de contextualizarlos.

- La asignación conjunta y concertada de lugares de fácil identificación para los niños y las niñas, con el fin de que sus objetos personales estén a su alcance, contribuyendo al orden y al sentido de pertenencia.

Partiendo del anterior análisis, se asumen nuevos retos $y$ estrategias que favorezcan y den claridad a nivel municipal, académico y de comunidad frente al tema de espacios públicos, generando diferentes discursos y reflexiones frente al tema. La generación de ambientes seguros, protectores y potenciadores en la educación inicial son, en gran medida, una responsabilidad compartida que involucra a todos los actores sociales. Por lo tanto, se requieren reflexiones y espacios de encuentro con diferentes agentes educativos a nivel político, educativo, religioso y cultural.

\section{CONCLUSIONES Y DISCUSIÓN}

En el abordaje de la educación motriz y de sus aportes a la salud, se destaca que la frecuencia semanal es solo de dos veces por semana, siendo más común el abordaje de este elemento en forma transversal en las diferentes jornadas de la cotidianidad escolar.
Esto puede llegar a tener efectos no muy favorables en la adquisición de mejoras en el desarrollo de las habilidades motrices básicas y de hábitos de salud, pues la etapa escolar es un gran momento para la promoción de hábitos de salud como la higiene, la alimentación y el sueño (Aliño Santiago, Navarro Fernández, López Esquirol, \& Pérez Sánchez, 2007); por lo tanto, la escuela debe garantizar la apropiación de estos elementos a partir del abordaje de los mismos durante una buena parte de la jornada escolar. Entre las acciones que se proponen, se encuentran:

Fomentar hábitos bucodentales saludables, el baño diario, el lavado de manos antes de la ingestión de las comidas y después de la defecación, la limpieza y corte de las uñas, la responsabilidad con el aseo anal y genital cuando están en condiciones de hacerlo (Aliño Santiago et al., 2007, p. 4).

En concordancia con lo anterior, el aula o salón de clase es el espacio predilecto del abordaje de lo motriz en los preescolares del municipio de Bello. Las canchas deportivas y las zonas verdes son los sitios menos utilizados por las profesoras para el trabajo de lo motriz con respecto a la salud, situación que en la mayoría de los casos se presenta porque la institución carece de dichos escenarios, lo que posiblemente limita las posibilidades de un desarrollo motor adecuado, y en consecuencia puede llegar a mitigar, en parte, su desarrollo físico e integral, pues se supone que los niños y las niñas (al terminar el nivel preescolar) deben estar preparados física, psicológica y socialmente para iniciar a la escuela regular y cumplir así con los nuevos retos y tareas asignadas (Aliño Santiago et al., 2007). Frente a la importancia del espacio, se considera que:

La organización espacial propicia que se den unas $u$ otras experiencias. Pensar en un espacio que dé respuesta a las necesidades de los niños y de los 
adultos que los acompañamos en su desarrollo, supone organizarlo de tal manera que propicie múltiples relaciones y situaciones de aprendizaje a la vez que ayude a la adquisición de autonomía. Bajo este marco nos planteamos un espacio estable que permita a las criaturas ir construyendo una imagen ordenada del mundo que las rodea, pero también abierto a la flexibilidad y variedad para permitir satisfacer las distintas y cambiantes necesidades que acontecen en la escuela. De la misma forma, los espacios deben ser sanos y seguros, evitando riesgos innecesarios sin caer en la sobreprotección, apoyando el uso y disfrute de manera autónoma por parte de los niños, y en donde sea posible el acceso a todas las dependencias, los materiales y las personas (Osoro Sierra \& González del Río, 2008, p. 24) (2008, p. 24).

Los principales fines que persiguen los profesores en sus centros educativos en la educación motriz, se refiere a potenciar el ámbito motor y fomentar la integración del estudiante, lo que evidencia la importancia de las metas pedagógicas, no obstante, es posible que su consecución sea limitada por los recursos temporales y espaciales de las instituciones, expresado de la siguiente forma:

La educación física en la actualidad, aunque con más de medio siglo de retraso, ha ampliado sus responsabilidades para abarcar aquellas que incluyen desde potenciar las condiciones físicas básicas o de desarrollo de determinadas destrezas deportivas, hasta interesarse por aquellos otros objetivos que la comprensión unitaria del hombre le permite y le exige ahora: los ámbitos afectivo, cognitivo, tónicoemocional y simbólico (Gil Madrona,
Contreras Jordán, \& Gómez Barreto, 2008, p. 74).

Los preescolares cuentan, en su mayoría, con docentes de apoyo para enfrentar la educación motriz y coordinan su trabajo con otros compañeros de trabajo, no obstante, en solo una institución se evidencia que este apoyo es dado por un profesional del área de educación física, lo que va en contravía de la Ley 934, que establece que en todos los establecimientos educativos, privados y oficiales, conforme a la Ley 115 (Colombia. Congreso de la República, 1994), se incluirá el programa para el desarrollo de la educación física (Colombia. Congreso de la República, 2004, p. 1), lo que infortunadamente no se cumple en los preescolares del municipio de Bello.

La educación motriz es susceptible de ser abordada por las siete dimensiones que propone el Ministerio de Educación Nacional: socio-afectiva, corporal, cognitiva, comunicativa, estética, espiritual y ética, lo que ratifica que el abordaje de la educación preescolar y la integralidad, no solo es necesario sino posible (Colombia. Ministerio de Educación Nacional, 1998); no obstante, los profesores del municipio de Bello consideran que las dos dimensiones que más aportan a la educación motriz y a la salud, son la corporal y la cognitiva. La mayoría de profesores indagados manifiestan que, en general, no existen dificultades a la hora de abordar la educación motriz y la salud, pero algunos de ellos expresaron que en algunos casos presentan un excesivo número de estudiantes, pues en educación preescolar se sugieren grupos poco numerosos, ya que esto favorece el aprendizaje, especialmente si se trata de niños de bajo nivel socioeconómico (de la Barra, Toledo, \& Rodríguez, 2002).

La mayoría de profesores de preescolar del municipio de Bello poseen formación en educación superior, siendo el nivel de pregrado el más común; sin embargo, la formación 
posgradual es aún incipiente. Por otro lado, la presencia de practicantes como docentes o docentes de apoyo en los preescolares también es común. Frente a la experiencia profesional, la mayoría de profesores poseen más de 10 años de ella en la educación en general, pero también en el trabajo con niños y niñas. En su trabajo particular en los preescolares del municipio de Bello, los profesores se desempeñan en diferentes niveles: prejardín, jardín y transición; incluso, el primer año de educación básica, no obstante, el grado más común de desempeño es el de prejardín y el menos frecuente es primero de básica primaria.

A pesar de que el cuestionario es muy útil para diagnosticar el abordaje de la educación motriz en función de la educación para la salud, tiene el inconveniente de que la información que ofrece depende de la posición del profesor indagado, lo que puede llegar a resultar un tanto subjetivo, como se describe en el diseño original (Sanz-Arazuri, Alonso-Ruiz, Valdemoros-SanEmeterio, \& Ponce de León, 2013).

\section{REFERENCIAS BIBLIOGRÁFICAS}

Abad Molina, J. (2014). Cabanellas, I. y Eslava, C. (2005). Territorios de la Infancia. Diálogos entre arquitectura y pedagogía. Barcelona: Graó. Revista Latinoamericana de Educación Infantil, 3(2), 267-269.

Aliño Santiago, M., Navarro Fernández, R., López Esquirol, J. R., \& Pérez Sánchez, I. (2007). La edad preescolar como momento singular del desarrollo humano. Revista Cubana de Pediatría, 79(4), 1-13.

Borja, J. (2003). La ciudad conquistada. Madrid, España: Alianza.

Caballenas, I., Eslava, C., Fornasa, W.,
Hoyuelos, A., Polonio, R., \& Tejada, M. (2005). Territorios de la infancia: diálogos entre arquitectura y pedagogía. Barcelona, España: Graó.

Colombia. Asamblea Nacional Constituyente. (1991). Constitución Política de Colombia. Bogotá, Colombia: República de Colombia.

Colombia. Congreso de la República. (1994). Ley 115 de 1994: por la cual se expide la ley general de educación. Bogotá, Colombia: Diario Oficial.

Colombia. Congreso de la República. (2004). Ley 934 de 2004: por la cual se oficializa la Política de Desarrollo Nacional de la Educación Física y se dictan otras disposiciones. Bogotá, Colombia: Diario Oficial.

Colombia. Ministerio de Educación Nacional. (1998). Serie lineamientos curriculares: preescolar. Bogotá, Colombia: Ministerio de Educación Nacional.

Colombia. Ministerio de Educación Nacional. (2009). Desarrollo infantil y competencias en la primera infancia. Bogotá, Colombia: Ministerio de Educación Nacional.

Colombia. Ministerio de Educación Nacional. (2014). Seguimiento al desarrollo integral de las niñas y los niños en la educación inicial. Bogotá, Colombia: Ministerio de Educación Nacional.

Colombia. Ministerio de Educación Nacional. (2017). Bases curriculares para la educación inicial y preescolar. Bogotá, Colombia: Ministerio de Educación Nacional. 
de la Barra, F., Toledo, V., \& Rodríguez, J. (2002). Estudio de salud mental en dos cohortes de niños escolares de Santiago occidente: II: Factores de riesgo familiares y escolares. Revista Chilena de NeuroPsiquiatría, 40(4), 347-360. https://doi. org/10.4067/S0717-92272002000400007

Fondo de las Naciones Unidas para la Infancia [UNICEF]. (2019). Ambientes protectores y oportunidades para toda la infancia. Retrieved from https://uni.cf/2TLtTZN

García Vázquez, M. (2013). Propuesta de anteproyecto para la recuperación del sector 4 de Bogotá. (Tesis Arquitectura). Universidad Nacional Autónoma de México, Facultad de Arquitectura, Ciudad de México.

Gil Madrona, P., Contreras Jordán, O. R., \& Gómez Barreto, I. M. (2008). Habilidades motrices en la infancia y su desarrollo desde una educación física animada. Revista Iberoamericana de Educación, 47(1), 71-96.

Malone, K. (2010). Dando a los niños libertad para desarrollar ciudades idóneas para la infancia. Espacio Para La Infancia, (34), 20-25.

Osoro Sierra, J. M., \& González del Río, O. M. (2008). Escenarios para el análisis y la construcción de un modelo de educación infantil. Revista Iberoamericana de Educación, 47(1), 15-32.

Pinheiro de Almeida, M. T. (2012). El jugar de los niños en el espacio público. (Tesis Doctoral). Universitat de Barcelona, Departamento de Didáctica y Organización Educativa, Barcelona.
Román Rivas, M., \& Pernas Riaño, B. (2009). ¡Hagan sitio, por favor!: la reintroducción de la infancia en la ciudad. Madrid, España: Ministerio de Medio Ambiente y Medio Rural y Marino.

Sánchez Parga, J. (2004). Orfandades infantiles y adolescentes: introducción a una sociología de la infancia. Quito, Ecuador: Abya-Yala, Universidad Politécnica Salesiana.

Sanz-Arazuri, E., Alonso-Ruiz, R. A., Valdemoros-San-Emeterio, M. Á., \& Ponce de León, A. (2013). Validación de un cuestionario que analiza cómo trabaja el profesorado de la etapa infantil la educación para la salud desde el ámbito motor. Revista Iberoamericana de Diagnóstico Y Evaluación - E Avaliação Psicológica, 1(35), 9-34.

Tonucci, F. (2015). La ciudad de los niños. Barcelona, España: Graó.

Vertel Betancur, L., \& Cuervo Duque, L. (2013). ¡A fortalecer ambientes protectores para la niñez! Crianza \& Salud, 12(1105). 